\title{
Can Contrast-Enhanced Multi-Detector Computed Tomography Replace Transesophageal Echocardiography for the Detection of Thrombogenic Milieu and Thrombi in the Left Atrial Appendage: A Prospective Study with 124 Patients
}

\section{Kann die Kontrastmittel-verstärkte Mehrzeilen-Computertomografie die trans- ösophageale Echokardiografie bei der Detektion von thrombogenem Milieu und Vorhofohrthromben ersetzen? Eine prospektive Studie mit 124 Patienten}

\author{
Authors \\ R. Homsi ${ }^{1}$, B. Nath ${ }^{2}$, J. A. Luetkens' ${ }^{1}$, J. O. Schwab ${ }^{3}$, H. H. Schild ${ }^{1}$, C. P. Naehle ${ }^{1}$ \\ Affiliations \\ Department of Radiology, University of Bonn, Germany \\ Department of Medicine I - Cardiology, SHG-Kliniken Völklingen, Germany \\ Department of Medicine - Cardiology, University of Bonn, Germany
}

\author{
Key words \\ - echocardiography \\ - cardiac \\ - CT \\ - left atrial appendage \\ - thrombus
}

\begin{abstract}
$\nabla$

Purpose: To assess the diagnostic value of contrast-enhanced multi-detector computed tomography (MD-CT) for identifying patients with left atrial appendage (LAA) thrombus or circulatory stasis.
\end{abstract}

Materials and Methods: 124 patients with a history of atrial fibrillation and/or cerebral ischemia ( 83 men, mean age $58.6 \pm 12.4$ years) and with a clinical indication for MD-CT of the heart and for transesophageal echocardiography (TEE) were included in the study. LAA thrombus or thrombogenic milieu was visually identified in TEE and MD-CT. In addition, MDCT was analyzed quantitatively measuring the Hounsfield units (HU) of the left atrium (LA), the LAA and the ascending aorta (AA), and calculating the HU ratios LAA/AA (HU [LAA/AA]) und LAA/LA (HU [LAA/LA]). Sensitivity, specificity, negative predictive value (NPV) and positive predictive value (PPV) were calculated.

received 28.1.2015 accepted 26.7.2015

Bibliography DOI http://dx.doi.org/ 10.1055/s-0041-106067 Published online: 30.9.2015 Fortschr Röntgenstr 2016; 188 : 45-52 @ Georg Thieme Verlag KG Stuttgart · New York · ISSN 1438-9029

\section{Correspondence} Dr. Rami Homsi

Radiology, University Hospital Bonn

Sigmund-Freud-Straße 25

53127 Bonn

Germany

Tel.: ++ 49/228/28719630

Fax: ++49/2 28/28715598

Rami.Homsi@ukb.uni-bonn.de tional calculation of $\mathrm{HU}$ ratios did not improve the diagnostic performance of MD-CT.

Key Points:

- MD-CT can reliably exclude atrial appendage thrombi/thrombogenic milieu.

- MD-CT is an alternative method in patients with contraindications to TEE.

- Calculation of relative HU ratios does not improve the diagnostic value of MD-CT.

Citation Format:

- Homsi R, Nath B, Luetkens JA etal. Can Contrast-Enhanced Multi-Detector Computed Tomography Replace Transesophageal Echocardiography for the Detection of Thrombogenic Milieu and Thrombi in the Left Atrial Appendage: A Prospective Study with 124 Patients. Fortschr Röntgenstr 2016; 188: 45-52

\section{Zusammenfassung \\ $\nabla$}

Ziel: Die Bestimmung der diagnostischen Aussagekraft der kontrastmittelverstärkten Computertomografie (MD-CT) hinsichtlich der Beurteilung des linken Vorhofs (LA) und des linken Vorhofohrs (LAA) zur Detektion von Thromben oder eines hohen thrombogenen Milieus.

Material und Methoden: Es wurden 124 Patienten mit Vorhofflimmern und/oder zerebraler Ischämie (83 Männer, Durchschnittsalter 58,6 $\pm 12,4$ Jahre) eingeschlossen bei denen die Indikation zur Durchführung einer transösophagealen Echokardiografie (TEE) und zusätzlich einer MD-CT des Herzens bestand. Ein LAA-Thrombus oder hohes thrombogenes Milieu wurden visuell mittels TEE und MD-CT beurteilt. Zusätzlich wurden die MD-CTs quantitativ über die Bestimmung der mittleren Röntgendichte in LA, LAA und Aorta ascendens (AA) und der berechneten relativen Röntgendichte LAA/AA (rD [LAA/AA]) und LAA/LA (rD [LAA/LA]) analysiert. Sensitivität, Spezifität, nega- 
tiv prädiktiver Wert (NPV) und positiv prädiktiver Wert (PPV) wurden berechnet.

Ergebnisse: Die Prävalenz eines Thrombus oder eines hohen thrombogenen Milieus im TEE betrug 21,8\%. Die rD waren bei Patienten mit Thrombus oder hohem thrombogenen Milieu erniedrigt ( $\mathrm{rD}$ [LAA/AA]: $0,590 \pm 0,248$ vs. $0,909 \pm 0,141 ; \mathrm{p}<0,001$ und $\mathrm{rD}$ [LAA/LA] 0,689 $\pm 0,366$ vs. 1,082 $\pm 0,228 ; \mathrm{p}<0,001)$. Die visuelle Bildanalyse der MD-CT für die Diagnose eines Thrombus oder eines hohen thrombogenen Milieus ergab verglichen mit der TEE eine Sensitivität von $81,5 \%$, eine Spezifität von 96,9\%, einen PPV von $87,5 \%$ und einen NPV von 95,2\%. Durch die Kombination von visueller und quantitativer Analyse, wobei eines der beiden Kriterien positiv sein musste, verschlechterte sich die Sensitivität auf $77,8 \%$, die Spezifität auf $91,8 \%$, der PPV auf $72,4 \%$ und der NPV auf $94,9 \%$.

Schlussfolgerung: Die visuelle Beurteilung der MD-CT weist mit 95,1\% einen hohen NPV auf und stellt bei Patienten mit einer Kontraindikation für die Durchführung einer TEE eine diagnostische Alternative dar. Die zusätzliche Berechnung relativer Dichtewerte verbessert die diagnostische Aussagekraft der MD-CT nicht.

Kernaussagen:

- Die MD-CT kann LAA-Thromben und thrombogenes Milieu mit hoher Sicherheit ausschließen.

- Bei TEE-Kontraindikationen kann die MD-CT als alternative Untersuchungsmethode eingesetzt werden.

- Die Bestimmung relativer Dichtewerte verbessert die Detektion von Thromben/thrombogenem Milieu nicht.

\section{Introduction}

$\nabla$

Cardiogenic embolisms are one of the most common causes of ischemic stroke and transitory ischemic attack (TIA). Atrial fibrillation accounts for for $60 \%$ of all cardioembolic strokes [1]. A therapy approach here is the conversion of atrial fibrillation into sinus rhythm (rhythm control), e.g. by medication-based or electrical cardioversion or by pulmonary vein ablation. However, thrombi in the left atrial appendage are a contraindication for such a procedure for restoring rhythm [2] since this can lead to cerebral embolization due to the restored atrial contraction and the normalization of flow conditions [3].

The gold standard for ruling out an atrial thrombus is transesophageal echocardiography (TEE), but there are contraindications for this procedure such as esophageal pathologies (recent esophageal surgery or bleeding) [4]. Therefore, an alternative examination technique for evaluating the left atrium (LA) and the left atrial appendage (LAA) is desirable. Contrast-enhanced multidetector computed tomography (MD-CT) is a possible alternative for the evaluation of LA morphology and for the detection of an LAA thrombus since sufficient temporal and spatial resolution can be achieved with this technique. Up to date only a few studies described the diagnostic value of MD-CT for LA/LAA evaluation and it is noteworthy that the sensitivity $(29-100 \%)$ and specificity (64-98\%) vary greatly in the different studies [5 - 8].

The purpose of this study was therefore to determine the diagnostic accuracy of MD-CT for detecting thrombi or a high thrombogenic milieu via visual and quantitative evaluation of the LA and LAA.

\section{Materials and Methods}

$\nabla$

\section{Study population}

The study was approved by the responsible ethics committee. Patients with an indication for TEE and additionally for computed tomography of the heart (e. g. to rule out coronary artery disease prior to the start of long-term treatment with a class I antiarrhythmic agent or prior to pulmonary vein ablation for anatomy visualization) were prospectively included after providing written informed consent. The exclusion criteria were the presence of a contraindication for contrast-enhanced CT examination (known contrast agent or iodine allergy, thyroid disease), pregnancy, and an age of less than 18 years.

\section{Echocardiography}

Transthoracic echocardiography (TTE) and TEE were performed with a system V or VIVID 7 echocardiography system. TTE was performed with a $1.7 / 3.4 \mathrm{MHz}$ probe and TEE with a multiplane $5 \mathrm{MHz}$ probe (GE Medical Systems). All standard planes were examined and digitally stored. The atrial volume, left-ventricular ejection fraction (LVEF), and left-ventricular end-diastolic volume (LVEDV) were determined in TTE. The flow velocity in the left atrial appendage was determined in TEE using pulse wave Doppler (PW Doppler). To determine the spontaneous echo contrast (SEC) and to rule out thrombi, the LA and LAA were imaged on at least two planes. A thrombus in the LAA was defined as a circumscribed homogeneous and echogenic mass with a non-myocardial texture. The severity of the SEC was graded from I to IV ( I = minor homogeneous contrast enhancement; II = significant homogeneous contrast enhancement; III = significant, dense, and inhomogeneous, slow-moving contrast; IV = dense slow-moving contrast) [9].

\section{MD-CT \& reconstruction}

All MD-CT examinations were performed on an MD-CT unit with 64 rows (Philips Brilliance 64, detector collimation: $64 \times 0.625 \mathrm{~mm}$, tube current: $650 \mathrm{mAs} /$ rotation, tube voltage: $120 \mathrm{kV}$, primary reconstructed slice thickness: $0.9 \mathrm{~mm}$, increment: $0.45 \mathrm{~mm}$, rotation time $0.4 \mathrm{~s}$, pitch: 0.2 , retrospective ECG gating). A $90 \mathrm{ml}$ bolus of an iodine-containing contrast agent (Solutrast 370, Bracco Imaging, Germany) was injected intravenously with an injection rate of $6 \mathrm{ml} / \mathrm{s}$ followed by a saline bolus with an injection rate of $4 \mathrm{ml} / \mathrm{s}$. After detection of the contrast bolus (bolus track technique with 1 frame/second, threshold value of $150 \mathrm{HU}$ of the ascending aorta, measurement field at the level of the tracheal bifurcation), image acquisition was started with a time delay of 6 seconds. Patients were monitored during the entire examination using ECG and pulse oximeter. Systolic images (30\% of $\mathrm{RR}$ interval) and diastolic images (75\% of RR interval) were reconstructed for image analysis.

\section{Image analysis}

TEE was evaluated by two cardiologists who were blinded to the MD-CT result. The TEE examination was pathological if a thrombus or a high thrombogenic milieu (SEC grade III/ IV) was detected [9].

The MD-CT scans were evaluated by two radiologists blinded to the TEE result and were classified as normal (homogeneous contrast enhancement of the entire atrial appendage) 
or high thrombogenic milieu/thrombus (not completely visible atrial appendage due to inhomogeneous or irregular contrast enhancement) $[7,10]$. A CT examination was then classified as pathological (i.e., presence of an atrial appendage thrombus or a thrombogenic milieu) when one of the two cardiac phases showed a pathological finding. The quantitative $\mathrm{HU}$ values were determined using a circular region-of-interest in the center of the LA, in the LAA and in the AA. This allowed the calculation of the relative Hounsfield ratios $\mathrm{LAA} / \mathrm{AA}\left(\mathrm{HU}\left(\mathrm{LAA}_{\mathrm{AA}}^{\mathrm{AA}}\right)\right)$ and $\mathrm{LAA} / \mathrm{LA}\left(\mathrm{HU}\left(\mathrm{LAA}_{\mathrm{LA}}\right)\right)[5]$.

\section{Statistics}

Statistical evaluation was performed using Analyse-it for Microsoft Excel (Version 3.76.1.2, Analyse-it Software, Ltd.; http://www.analyse-it.com/). Categorical data is provided as number and percent, and continuous data as average and standard deviations. Categorical groups were compared using the Pearson's chi-square test, and the continuous data of different groups was compared using the WilcoxonMann-Whitney test. Correlations of continuous data were calculated with the Kendall test. With TEE as the gold standard, the sensitivity, specificity, negative predictive value (NPV), and positive predictive value (PPV) were calculated. The limit values for $\mathrm{HU}\left(\mathrm{LA}_{\mathrm{LAA}}\right)$ and $\mathrm{HU}\left({ }^{\mathrm{LAA}} / \mathrm{LA}\right)$ were determined on the basis of the receiver operating characteristic (ROC) curve.

\section{Results}

Of 137 patients, 13 patients did not agree to participate in the study and 2 of these patients refused all further diagnostics and treatment. In total, 124 patients ( 83 men, 41 women, average age $58.6 \pm 12.4$ years) were prospectively included in the study ( $($ Table 1$)$. TEE was performed as part of the diagnostic work-up for atrial fibrillation (e.g., prior to cardioversion or pulmonary vein ablation) in 117 (94\%) patients. In the remaining patients TEE was performed as part of the search for embolic sources due to a stroke or a TIA. The average duration between TEE and MD-CT was $1.16 \pm 1.38$ days.

\begin{tabular}{|l|l|}
\hline Table 1 Epidemiologic and clinical characteristics of the patients. \\
\hline age [in years] & $58.6 \pm 12.4$ \\
\hline men & $83(67 \%)$ \\
\hline BMI [kg/m² body surface] & $27.4 \pm 3.9$ \\
\hline heart rate (TEE) & $71.0 \pm 10.8$ \\
\hline heart rate (MD-CT) & $68.8 \pm 10.4$ \\
\hline atrial fibrillation & $117(94 \%)$ \\
\hline hypertension & $95(77 \%)$ \\
\hline known coronary heart disease & $20(16 \%)$ \\
\hline ASD & $13(10.5 \%)$ \\
\hline PFO & $20(16 \%)$ \\
\hline age $>75$ years & $9(7 \%)$ \\
\hline diabetes mellitus & $10(8 \%)$ \\
\hline patient with thrombus or & $27(22 \%)$ \\
\hline thrombogenic milieu & 17 men: $63 \%$ \\
\hline
\end{tabular}

BMI: Body Mass Index; CAD: coronary artery disease; ASD: atrial septal defect; PFO: persistent foramen ovale; TEE: transesophageal echocardiography; MD-CT: multi-detector computed tomography; \pm standarddeviation.
The CT examinations had a median CTDI of $38.2 \mathrm{mGy}$, a median dose-length product of $887.50 \mathrm{mGycm}$ (IQR 111.02 $\mathrm{mGycm}$ ) and a calculated median total dose of $11.8 \mathrm{mSv}$ (IQR $1.5 \mathrm{mSv}$ ). These values correspond with the results of the protection-I study of Hausleiter et al. [11].

The prevalence of a pathological finding (thrombus or high thrombogenic milieu) in TEE was $21.8 \%$ in 17 (63\%) men and $10(37 \%)$ women. Patients with a thrombus or a high thrombogenic milieu were significantly older $(66.9 \pm 11.0$ years vs. $56.4 \pm 11.8$ years; $\mathrm{p}<0.001$ ), and more frequently had hypertension ( $92.3 \%$ vs. $73.2 \%$; $=0.039$ ), coronary artery disease ( $38.5 \%$ vs. $10.9 \%$; $<0.001$ ) and a lower LVEF ( $50.5 \% \pm 9.4 \%$ vs. $61.8 \% \pm 20.2 \% ; \mathrm{p}=0.034$ ). The comparison of the clinical characteristics by the presence of a thrombus or a high thrombogenic milieu is summarized in $\bullet$ Table 2.

The relative HU ratios were lower in patients with a pathological finding $\left(\mathrm{HU}\left(\mathrm{LAA}_{\mathrm{AAA}}\right): 0.590 \pm 0.248\right.$ vs. $0.909 \pm 0.141$; $\mathrm{p}<0.001$ und $\mathrm{HU}\left(\mathrm{LAA}^{\mathrm{LAA}} / \mathrm{LA}\right) \quad 0.689 \pm 0.366$ vs. $1.082 \pm 0.228$; $\mathrm{p}<0.001)$. The presence of a thrombus in TEE was associated with a lower LAA flow velocity in TEE $(0.278 \mathrm{~m} / \mathrm{s}$ vs. $0.575 \mathrm{~m} /$ $\mathrm{s} ; \mathrm{p}<0.001)$. There were only marginal correlations between the LAA flow velocity and $\mathrm{HU}\left({ }^{\mathrm{LAA}} / \mathrm{AA}\right)(\mathrm{r}=0.335 ; \mathrm{p}=0.002)$ and $\mathrm{HU}\left(\mathrm{LA}_{\mathrm{LA}}\right)(\mathrm{r}=0.274 ; \mathrm{p}=0.009)$. 0 Table 3 summarizes the results from the comparison of the relative HU ratios, the LAA flow velocity, and the LA volume by the presence of a thrombus or a high thrombogenic milieu in TEE.

Compared to TEE, visual image analysis for the diagnosis of a thrombus or a high thrombogenic milieu in MD-CT yielded a sensitivity of $81.5 \%$, a specificity of $96.9 \%$, a positive predictive value (PPV) of $87.5 \%$ and a negative predictive value (NPV) of $95.2 \%$. For the diagnosis of an LAA thrombus, the sensitivity was $72.2 \%$, the specificity was $96.2 \%$, the PPV was $83.6 \%$, and the NPV was $92.9 \%$.

The ROC analysis ( $\bullet$ Fig. 1 ) of the absolute HU ratio in LAA yielded an area under the curve (AUC) of 0.829 (95\% confidence interval (CI) $0.730-0.928), \mathrm{HU}\left({ }^{\mathrm{LAA}} / \mathrm{LA}\right)$ yielded an AUC of $0.829(95 \% \mathrm{CI} 0.714-0.944)$ and $\mathrm{HU}\left(\mathrm{LAA}_{\mathrm{AA}}\right)$ an AUC of $0.816(95 \% \mathrm{Cl} 0.702-0.930)$. To examine whether the inclusion of a quantitative analysis improves diagnostic significance, a combination of visual and quantitative evaluation was defined. This combined criterion was positive if the visual analysis or the quantitative analysis was positive for the presence of a thrombus or a high thrombogenic milieu (SEC $\mathrm{III} / \mathrm{IV}) . \mathrm{HU}(\mathrm{LAA} / \mathrm{LA})$ was selected as a parameter for quantitative analysis since this parameter has the greatest AUC among parameters with a significant difference between patients without and with a thrombus or thrombogenic milieu ( $\odot$ Table 2). 0.75 was defined as the upper limit for $\mathrm{HU}\left(\mathrm{LAA}^{\mathrm{LAA}} / \mathrm{L}\right)$ based on the ROC analyses ( $\bullet$ Fig. 2 ). This combined score did not improve diagnostic significance: sensitivity worsened to $77.8 \%$, specificity to $91.8 \%$, PPV to $72.4 \%$, and NPV to $94.9 \%$ under consideration of $\mathrm{HU}(\mathrm{LAA} / \mathrm{LA})$.

\section{Discussion \\ $\nabla$}

In this study it was shown that MD-CT may be used as a noninvasive examination technique for detecting thrombi and a high thrombogenic milieu in the left atrial appendage. While a sensitivity of $93.3-100 \%$, a specificity of $99-100 \%$, a PPV of $86-100 \%$, and a NPV of $98.9-100 \%$ were reported for TEE $[12,13]$, MD-CT showed a sensitivity of $81.5 \%$, a spe- 


\begin{tabular}{|llll|}
\hline & $\begin{array}{l}\text { thrombus/high } \\
\text { thrombogenic milieu }\end{array}$ & $\begin{array}{l}\text { no thrombus/no high } \\
\text { thrombogenic milieu }\end{array}$ & p-value \\
\hline men & $17(63.0 \%)$ & $66(68.0 \%)$ & 0.331 \\
\hline women & $10(37.0 \%)$ & $31(32.0 \%)$ & 0.669 \\
\hline age [in years] & $66.9 \pm 11.0$ & $56.4 \pm 11.8$ & $<0.001$ \\
\hline BMI [kg/m² body surface] & $27.6 \pm 4.4$ & $27.30 \pm 3.80$ & 0.771 \\
\hline hypertension & $24(88.9 \%)$ & $71(73.20 \%)$ & 0.039 \\
\hline smoker & $1(3.7 \%)$ & $10(10.30 \%)$ & 0.311 \\
\hline diabetes mellitus & $4(14.8 \%)$ & $6(6.20 \%)$ & 0.111 \\
\hline coronary artery disease & $10(37.0 \%)$ & $10(10.30 \%)$ & $<0.010$ \\
\hline oral anticoagulation (marcumar) & $25(92.6 \%)$ & $88(90.70 \%)$ & 0.810 \\
\hline stroke & $4(14.8 \%)$ & $9(9.30 \%)$ & 0.810 \\
\hline atrial fibrillation & $22(81.5 \%)$ & $95(99.70 \%)$ & 0.378 \\
\hline BP systolic [mmHg] & $124.8 \pm 20.6$ & $124.10 \pm 16.80$ & 0.995 \\
\hline BP diastolic [mmHg] & $78.3 \pm 10.3$ & $77 \pm 10.60$ & 0.832 \\
\hline LVEF [\%] & $50.5 \pm 20.2 \%$ & $61.80 \pm 9.40 \%$ & 0.044 \\
\hline LAA flow velocity [m/s] & $0.28 \pm 0.05$ & $0.58 \pm 0.07$ & $<0.001$ \\
\hline LA volume [ml] & $82.2 \pm 30.2$ & $75.40 \pm 23.50$ & 0.541 \\
\hline LVEDV [ml] & $100.6 \pm 52.8$ & $92.80 \pm 35.50$ & 0.509 \\
\hline creatinine [mg/dl] & $1.11 \pm 0.32$ & $0.95 \pm 0.19$ & 0.044 \\
\hline HU ratio LAA [HU] & $180.9 \pm 98.7$ & $310.20 \pm 86.00$ & $<0.001$ \\
\hline HU ratio LA [HU] & $274.3 \pm 74.7$ & $291.70 \pm 81.50$ & 0.212 \\
\hline HU ratio AA [HU] & $313.3 \pm 84.8$ & $340.50 \pm 81.40$ & 0.123 \\
\hline HU(LAA/AA) & $0.590 \pm 0.284$ & $0.909 \pm 0.141$ & $<0.001$ \\
\hline HU(LAA/LA) & $0.689 \pm 0.366$ & $1.082 \pm 0.228$ & $<0.001$ \\
\hline BM:Body & & & \\
\hline
\end{tabular}

Table 2 Comparison of clinical characteristics by presence of a thrombus or thrombogenic milieu.

BMI: Body Mass Index; BP: blood pressure; LVEF: left-ventricular ejection-fraction; LAA: left atrial appendage; LA: left atrium; LVEDV: left-ventricular enddiastolic volume; AA: Aorta ascendens; rD: HU-ratio; mean \pm standard deviation.

Table 3 Comparison of Hounsfield-ratio (HU-ratio), LAA-flow velocity $(\mathrm{m} / \mathrm{sec}$ ) and LA-Volume (in $\mathrm{ml}$ ) by presence of a thrombus or a thrombogenic milieu in TEE.

\begin{tabular}{llllll} 
& number & HU(LAA/AO) & HU(LAA/LA) & LAA flow velocity & LA volume \\
\hline $\begin{array}{l}\text { thrombus or high thrombo- } \\
\text { genic milieu in TEE }\end{array}$ & 27 & $0.590 \pm 0.284$ & $0.689 \pm 0.366$ & $0.278 \pm 0.045$ & $82.2 \pm 30.2$ \\
$\begin{array}{l}\text { no thrombus or high throm- } \\
\text { bogenic milieu in TEE }\end{array}$ & 97 & $0.909 \pm 0.141$ & $1.082 \pm 0.228$ & $0.575 \pm 0.070$ & $75.4 \pm 23.5$ \\
\hline $\begin{array}{l}\text { P-value } \\
\text { nat }\end{array}$ & $<0.001$ & $<0.001$ & $<0.001$ & 0.514
\end{tabular}

TEE: transesophageal echocardiography; HU: HU-ratio; LAA: left atrial appendage; LA: left atrium; mean \pm standard deviation.

cificity of $96.9 \%$, a PPV of $87.5 \%$, and a NPV of $95.2 \%$ for visual evaluation with respect to a thrombus or a high thrombogenic milieu and a slightly lower sensitivity (72.2\%), specificity (96.2\%), PPV (83.6\%), and NPV (92.8\%) regarding only a LAA thrombus. This may be explained by a changed contrast distribution in the atrium and by a possibly incomplete homogeneous contrast enhancement in the atrial appendage due to the pathological atrial contraction in atrial fibrillation which may last up to 4 weeks after spontaneous cardioversion to a sinus rhythm [14].

Although MD-CT is already indicated in numerous cardiac diseases, it is at this time not recommended for the detection of LAA thrombi [15]. A recently published meta-analysis of 9 studies was only able to show a sensitivity of $81 \%$ (95\% CI; $70-90 \%)$ and a specificity of $90 \%$ (95\% CI $88-$ $91 \%$ ) [8]. A reason for this lower diagnostic performance compared to TEE may be the divergent results of the underlying studies $[8,10,16,17]$ in which the sensitivity was between $29 \%$ and $100 \%$ and the specificity was between $64 \%$ and $98 \%$. Possible reasons for this considerable variation in study results are methodological differences in the evaluation of the CT examinations (purely visual vs. purely quanti- tative vs. combined visual-quantitative), in the CT examination technique (ECG-triggered vs. not ECG-triggered) and in the contrast injection protocol (monophasic vs. biphasic). Finally, it must be noted that TEE, despite being the gold standard, is a method that is dependent on the examiner's experience and therefore does not have perfect sensitivity and specificity $[12,13]$.

The flow velocity in the left atrial appendage is an important echocardiographic marker for a thrombogenic milieu [9]. Probably due to the reduced atrial contractility and the resulting lower LAA flow velocities, the activation of the thrombogenic cascade with activation of prothrombotic factors and an increased fibrinogen level cause aggregation of red blood cells that can then be seen as spontaneous echocontrast in TEE [18]. 0 Fig. 3, 4 show the presence of SEC in TEE and MD-CT as examples. Spontaneous echocontrast is associated with an increased risk of thromboembolic events [9]. $\mathrm{HU}\left(\mathrm{LAA}_{\mathrm{AA}}\right), \mathrm{HU}(\mathrm{LAA} / \mathrm{LA})$, and LAA flow velocity differ significantly between patients without and with a thrombus or thrombogenic milieu ( $\bullet$ Table 3 ). This can be explained primarily by the incomplete mixing or delayed wash-in of the contrast agent in the LAA lumen [5] as part 
of the above-mentioned rheological changes. Based on the pathophysiology of thrombus formation, the quantification and consideration of the LA and LAA flow dynamics in MDCT therefore appeared promising. However, the diagnostic performance worsened when using the two best parameters $\mathrm{HU}\left(\mathrm{LAA}_{\mathrm{AA}}\right)$ and $\mathrm{HU}\left(\mathrm{LAA}_{\mathrm{LA}}\right)$ compared to visual evaluation alone. A possible explanation for this is the weak correlation of $\mathrm{HU}\left({ }^{\mathrm{LAA}} / \mathrm{AA}\right)$ and $\mathrm{HU}\left({ }^{\mathrm{LAA}} / \mathrm{LA}_{\mathrm{AA}}\right)$ with the echocardiographically determined flow velocities in the left atrial appendage $(r=0.335$ with $p=0.0015$ or $r=0.274$, $\mathrm{p}=0.0086$ ).

The reduced dignostic performance with respect to the relative $\mathrm{HU}$ ratio is in concordance to previous studies: Under consideration of the relative HU ratio, a study by Hur et al. showed a worsening of the sensitivity from $97 \%$ to

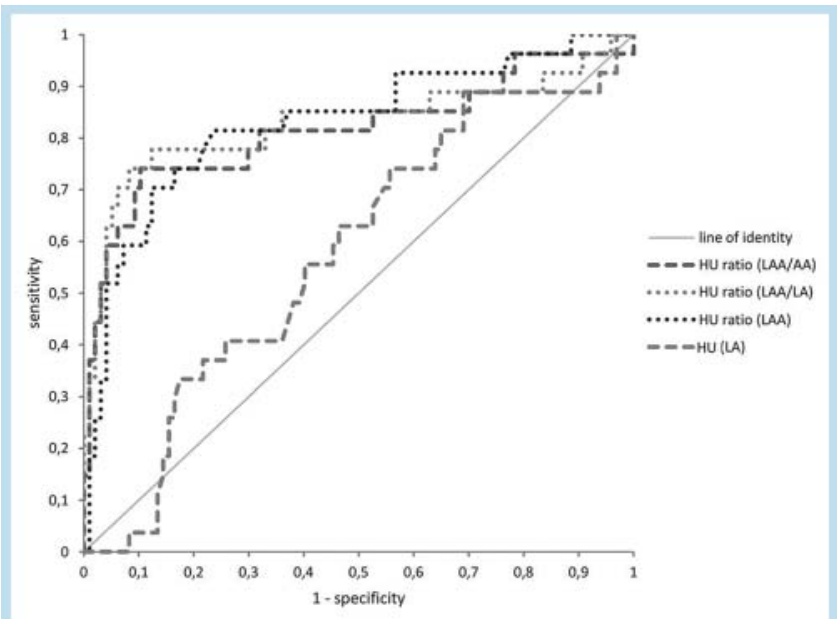

Fig. 1 Receiver operating characteristic (ROC) curve of the Hounsfield units in the left atrial appendage $(\mathrm{HU}(\mathrm{LAA}))$ and left atrium $(\mathrm{HU}(\mathrm{LA}))$ and the Hounsfield unit ratios $\mathrm{rD}(\mathrm{LAA} / \mathrm{AA})$ and $\mathrm{rD}(\mathrm{LAA} / \mathrm{LA})$. The area under the curve (AUC) for $r D(L A A / L A)$ is 0.829 . The $A U C$ for $r D(L A A / A A)$ is 0.815 (95\% Cl $0.701-0.930)$. (LAA: left atrial appendage; LA: left atrium; HU: Hounsfield units; rD: HU ratio).
$82 \%$ and of the specificity from $100 \%$ to $67 \%$ [10]. In a study by Shapiro et al., the sensitivity changed from $80 \%$ to $70 \%$, the specificity from $73 \%$ to $82 \%$, the PPV from $54 \%$ to $47 \%$ and the NPV from $90 \%$ to $92 \%$ [19]. However, other studies reported an additional diagnostic benefit from the use of relative $\mathrm{HU}$ ratios for the detection of thrombi $[20,21]$. Possible reasons for the difference in the influence of relative $\mathrm{HU}$ ratios on the diagnostic accuracy of MD-CT are the heart rate and rhythm at the time of MD-CT (sinus rhythm vs. atrial fibrillation), the type of scan triggering (fixed time interval vs. bolus tracking), the type of contrast media injection (e.g. monophasic, biphasic), use of ECG gating [17, 22], and the number of detector rows [23]. Heart rate and rhythm affect the stroke volume and thus also the cardiac output as a result of different prefilling of the left ventricle. Atrial fibrillation typically results in ineffective ventricular filling with a consecutively reduced cardiac output [24]. To minimize the effect of cardiac output on contrast wash-in, in this study the bolus track technique was used to start the CT examination. The window for measuring the intravascular $\mathrm{HU}$ ratio was placed in the ascending aorta. With this method the absolute $\mathrm{HU}$ ratios in the left atrium did not differ between patients with sinus rhythm and atrial fibrillation (270.8 \pm 70.8 vs. $274.4 \pm 81.2 ; \mathrm{p}=0.84)$. In contrast, $\mathrm{HU}\left(\mathrm{LAA}_{\mathrm{LA}}\right)$ showed a statistically significant difference between the two patient groups $(1.06 \pm 0.22$ vs. $0.92 \pm 0.34 ; \mathrm{p}=0.04)$. Nonetheless, the diagnostic performance of MD-CT was lower even under consideration of $\mathrm{HU}\left(\mathrm{LAA}_{\mathrm{LA}}\right)$. In the examined study population, $27.5 \%$ of patients without a thrombus or increased thrombogenic milieu had atrial fibrillation at the time of MD-CT. Since atrial fibrillation is associated with pathological atrial contractions and with changed contrast distribution in the atrium, the low prevalence in this group is a possible explanation for the worsening of the diagnostic significance of the quantitative analysis.

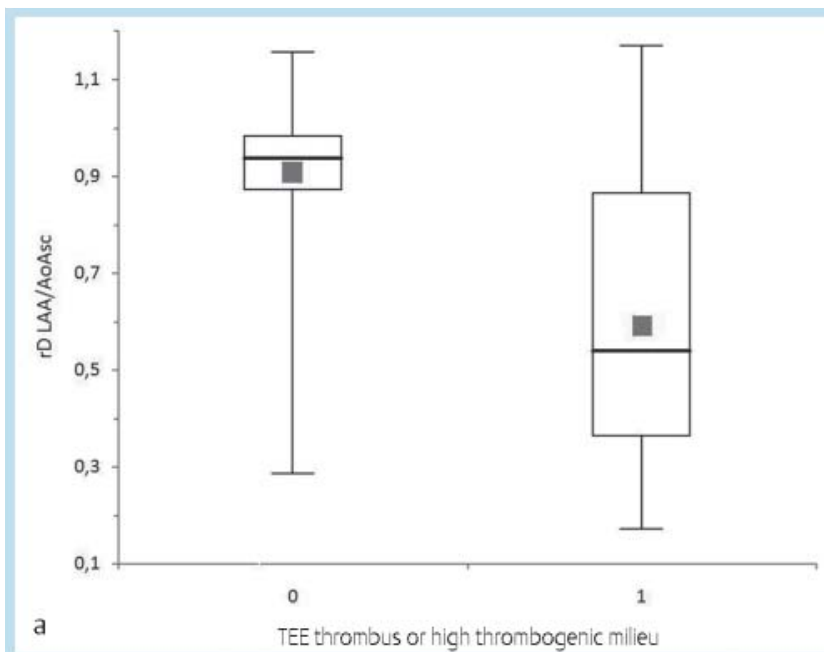

Fig. 2 Box-plots of the HU-ratio ( $\left.{ }^{\mathrm{LAA}} / \mathrm{AA}\right)$ a and the HU-ratio ( $\left.{ }^{\mathrm{LAA}} / \mathrm{LA}\right)$ b by presence of a thrombus or thrombogenic milieu. (rD: Hounsfield units ratio; TEE: transesophageal echocardiography; LAA: left atrial appendage; LA: left

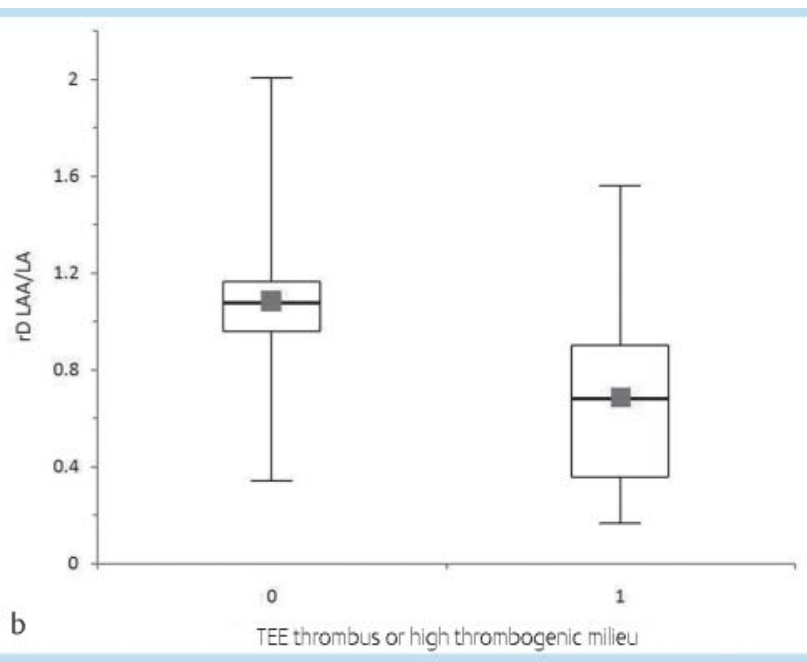

atrium; $\mathrm{HU}=$ Hounsfield-units; 1 : thrombus or thrombogenic milieu, 0 : no thrombus or thrombogenic milieu). 


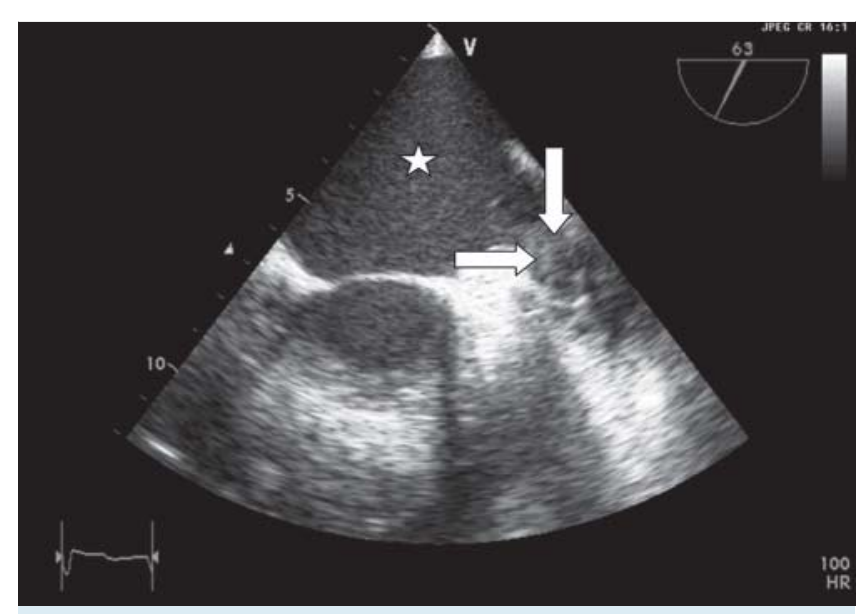

Fig. 3 Transesophageal echocardiography at an angle of $63^{\circ}$. The left atrium $\left({ }^{*}\right)$ and left atrial appendage which is occluded by a thrombus (arrows) can be seen.

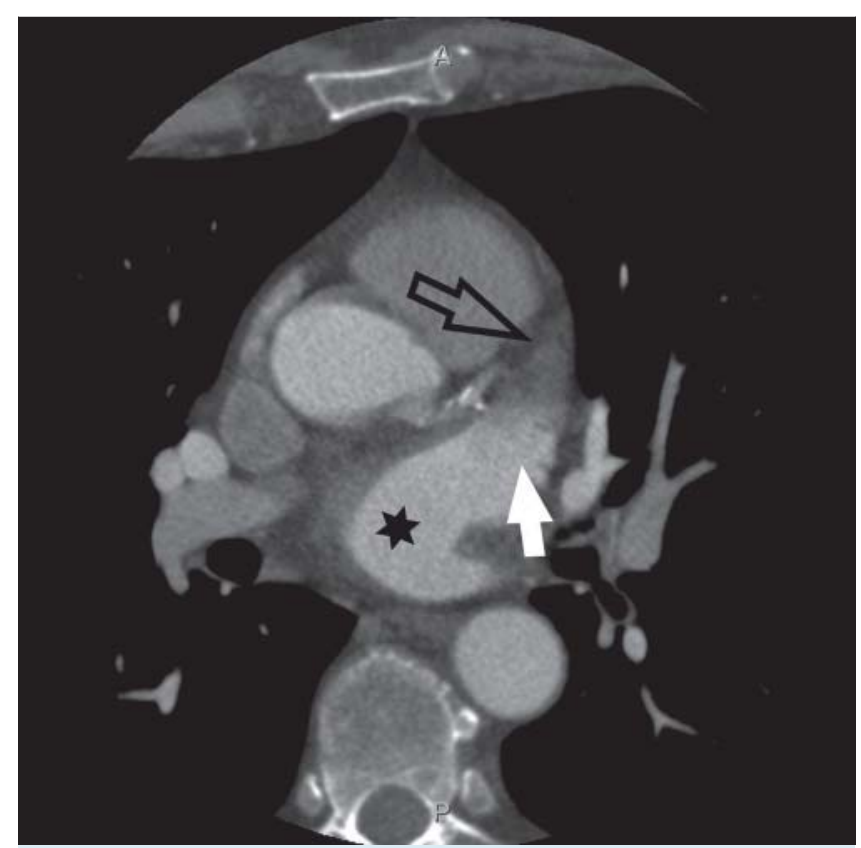

Fig. 4 Axial slice of contrast-enhanced multi-detector computed tomography. The left atrium $\left({ }^{*}\right.$ ) and left atrial appendage (LAA) can be seen. There is a thrombus in the LAA (black arrow) and a region of inhomogeneous contrast in the ostium of the LAA (white arrow) indicating slow flow.

A further possible explanation for the lower diagnostic significance with the addition of quantitative analysis may be the lower LVEF in the patient group with an increased thrombogenic milieu (LVEF $50.5 \%$ vs. 61.8\%). A reduced left venticular ejection fraction can result in delayed and changed contrast wash-in in the atrium due to the lower cardiac output and associated lower blood flow velocities [25].

The contrast media injection protocol can also affect the absolute and relative HU ratios due to the consequent variations in contrast wash-in, which may also explain differences in the diagnostic performance of the HU ratio between the left atrium, left atrial appendage, and ascend- ing aorta [26]. Although the contrast injection protocols in the various studies are similar to the one used in the present study, they may have contributed to changed contrast dynamics in connection due to differences in heart rhythm and rate. Finally, the use of ECG triggering also affects the diagnostic significance as a result of the improved image quality due to the better temporal resolution. Studies regarding the detection of atrial thrombi and a thrombogenic milieu that used ECG gating also showed a higher sensitivity and specificity than those without ECG gating or triggering $[5-8,17,22]$. For example, the sensitivity and specificity in a study without ECG triggering by Tang et al. were $36.4 \%$ and $93.7 \%$, respectively, the PPV was $28.6 \%$, and the NPV was $95.5 \%$ [17].

In our study 13 patients were diagnosed incorrectly (false negative or false positive). Possible explanations include prominent muscle tissue in the LAA (with misinterpretation as thrombus), de novo thrombi or thrombogenic milieu in the time interval between TEE and MD-CT, or image artifacts. Although the time interval between TEE and MDCT of $1.16 \pm 1.38$ days was very short, it cannot be ruled out that the thrombus or the thrombogenic milieu resolved during this time, (e. g. due to the start of anticoagulation therapy), thus resulting in a false-negative finding in MDCT. Atrial fibrillation during the examination as seen in $42 \%$ of patients in this study and the resulting artifacts can be misinterpreted as a filling defect and thus as a thrombus or thrombogenic milieu. Finally, like any other diagnostic test, TEE does not have perfect sensitivity (93.3-100\%) and specificity (99-100\%) even though it is the gold standard $[12,13]$.

An alternative with high diagnostic performance for the detection of LA/LAA thrombi is desirable particularly for patients in whom TEE is relatively (e.g. coagulopathy, recent gastrointestinal bleeding in the patient history) or absolutely (e.g. active esophageal bleeding) contraindicated [4]. A further noninvasive alternative without radiation exposure is magnetic resonance imaging (MRI) which allows visualization of the pulmonary vein anatomy and the LAA. Left-ventricular thrombi are routinely examined in MRI using the delayed enhancement technique. MRI is the gold standard particularly for apical thrombi [27]. The sensitivity of MRI for left-ventricular thrombi is significantly higher than that of TTE and TEE (MRI: $88 \%$, TTE: $23 \%$ and TEE: $40 \%$ ) with comparable specificity (>96\%) [28]. Thrombus detection via delayed enhancement could therefore play a role in the detection of LAA thrombi in the future. Other MRI examination techniques evaluated for the detection of LAA thrombi are black blood sequences, which, however, are susceptible to slow flow artifacts, and contrast-enhanced $2 \mathrm{D}$ and $3 \mathrm{D}$ perfusion sequences. The sensitivity and specificity are between $47 \%$ and $50 \%$ for these sequences $[29,30]$. Up to date, there are only a few studies regarding the use of MRI for the detection of LA/LAA thrombi. In the future MRI could play a greater role due to its noninvasiveness and the lack of radiation exposure. However, the known MRI contraindications must be taken into consideration. 


\section{Limitations}

$\nabla$

A limitation of the present study is the time interval between TEE and MD-CT that could not always be performed on the same day. Even if the time interval of $1.16 \pm 1.38$ days was short, it cannot be ruled out that a thrombus or thrombogenic milieu formed or resolved during this time interval.

\section{Conclusion}

The visual evaluation of MD-CT has a high negative predictive value of $95.1 \%$ and can be considered as an alternative diagnostic procedure particularly in patients with a contraindication for transesophageal echocardiography. The additional quantitative analysis of MD-CT with determination of relative $\mathrm{HU}$ ratios does not improve diagnostic significance.

\section{Clinical relevance}

1. In patients with cardiogenic embolism sources and a contraindication for TEE, MD-CT can be used as an alternative examination method.

2. Visual evaluation alone allows sufficiently reliable exclusion of thrombi of the atrial appendage or a thrombogenic milieu.

3. Determination of the relative HU ratios in addition to visual evaluation does not improve the diagnostic significance of MD-CT, thus making it possible to shorten the evaluation time.

4. The acquired $3 \mathrm{D}$ dataset additionally allows visualization of the pulmonary vein anatomy prior to catheter ablation.

\section{References}

1 Bonita R. Epidemiology of stroke. Lancet 1992; 339: 342 - 344

2 Camm AJ, Kirchhof P, Lip GY et al. Guidelines for the management of atrial fibrillation: the Task Force for the Management of Atrial Fibrillation of the European Society of Cardiology (ESC). Europace 2010; 12: $1360-1420$

3 Harrison MJ, Marshall J. Atrial fibrillation, TIAs and completed strokes. Stroke 1984; 15: $441-442$

4 Hilberath JN, Oakes DA, Shernan SK et al. Safety of transesophageal echocardiography. J Am Soc Echocardiogr 2010; 23: 1115-1127; quiz 1220-1111

5 Hur J, Kim YJ, Lee HJ et al. Left atrial appendage thrombi in stroke patients: detection with two-phase cardiac CT angiography versus transesophageal echocardiography. Radiology 2009; 251: 683-690

$6 \mathrm{Kim}$ YY, Klein AL, Halliburton SS et al. Left atrial appendage filling defects identified by multidetector computed tomography in patients undergoing radiofrequency pulmonary vein antral isolation: a comparison with transesophageal echocardiography. American heart journal 2007; 154: 1199-1205

7 Martinez MW, Kirsch J, Williamson EE et al. Utility of nongated multidetector computed tomography for detection of left atrial thrombus in patients undergoing catheter ablation of atrial fibrillation. JACC Cardiovascular imaging 2009; 2: 69-76

8 Wu X, Wang C, Zhang C et al. Computed tomography for detecting left atrial thrombus: a meta-analysis. Archives of medical science: AMS 2012; 8: $943-951$
9 Fatkin D, Kelly RP, Feneley MP. Relations between left atrial appendage blood flow velocity, spontaneous echocardiographic contrast and thromboembolic risk in vivo. Journal of the American College of Cardiology 1994; 23: 961 - 969

10 Hur J, Kim YJ, Lee HJ et al. Cardioembolic stroke: dual-energy cardiac CT for differentiation of left atrial appendage thrombus and circulatory stasis. Radiology 2012; 263: 688 - 695

11 Hausleiter J, Meyer T, Hermann F et al. Estimated radiation dose associated with cardiac CT angiography. JAMA 2009; 301: 500 - 507

12 Manning WJ, Weintraub RM, Waksmonski CA et al. Accuracy of transesophageal echocardiography for identifying left atrial thrombi. A prospective, intraoperative study. Ann Intern Med 1995; 123: 817-822

13 Hwang JJ, Chen JJ, Lin SC et al. Diagnostic accuracy of transesophageal echocardiography for detecting left atrial thrombi in patients with rheumatic heart disease having undergone mitral valve operations. The American journal of cardiology 1993; 72: 677-681

14 Manning WJ, Silverman DI, Katz SE et al. Impaired left atrial mechanical function after cardioversion: relation to the duration of atrial fibrillation. Journal of the American College of Cardiology 1994; 23: 1535 1540

15 Achenbach S, Barkhausen J, Beer M et al. Consensus recommendations of the German Radiology Society (DRG), the German Cardiac Society (DGK) and the German Society for Pediatric Cardiology (DGPK) on the use of cardiac imaging with computed tomography and magnetic resonance imaging. Fortschr Röntgenstr 2012; 184: 345 - 368

16 Dorenkamp $M$, Sohns C, Vollmann D et al. Detection of left atrial thrombus during routine diagnostic work-up prior to pulmonary vein isolation for atrial fibrillation: role of transesophageal echocardiography and multidetector computed tomography. International journal of cardiology 2013; 163: 26-33

17 Tang RB, Dong JZ, Zhang ZQ et al. Comparison of contrast enhanced 64slice computed tomography and transesophageal echocardiography in detection of left atrial thrombus in patients with atrial fibrillation. Journal of interventional cardiac electrophysiology: an international journal of arrhythmias and pacing 2008; 22: 199-203

18 Rastegar R, Harnick DJ, Weidemann P et al. Spontaneous echo contrast videodensity is flow-related and is dependent on the relative concentrations of fibrinogen and red blood cells. Journal of the American College of Cardiology 2003; 41: 603-610

19 Shapiro MD, Neilan TG, Jassal DS et al. Multidetector computed tomography for the detection of left atrial appendage thrombus: a comparative study with transesophageal echocardiography. Journal of computer assisted tomography 2007; 31: $905-909$

20 Staab W, Sohns C, Zwaka PA et al. Split-bolus single-phase cardiac multidetector computed tomography for reliable detection of left atrial thrombus: comparison to transesophageal echocardiography. Fortschr Röntgenstr 2014; 186: 1009-1015

21 Singh NK, Nallamothu N, Zuck VP et al. Left atrial appendage filling defects on 64-slice multidetector computed tomography in patients undergoing pulmonary vein isolation: predictors and comparison to transesophageal echocardiography. Journal of computer assisted tomography 2009; 33: 946-951

22 Monzen H, Shimoyama H, Hirata M et al. The optimal cardiac phase for detecting the thrombi of the left atrial appendage on multi-slice computed tomography in patients with atrial fibrillation. Radiol Phys Technol 2010; 3: $78-83$

23 Nishino M, Kubo T, Kataoka ML et al. Coronal reformations of the chest on 64-row multi-detector row CT: evaluation of image quality in comparison with 16-, 8- and 4-row multi-detector row CT. Eur J Radiol 2006; 59: $231-237$

24 Rodman T, Pastor BH, Figueroa W. Effect on cardiac output of conversion from atrial fibrillation to normal sinus mechanism. Am J Med 1966; 41: $249-258$

25 Handke $M$, Harloff $A$, Hetzel A et al. Left atrial appendage flow velocity as a quantitative surrogate parameter for thromboembolic risk: determinants and relationship to spontaneous echocontrast and thrombus formation - a transesophageal echocardiographic study in 500 patients with cerebral ischemia. J Am Soc Echocardiogr 2005; 18 : $1366-1372$

26 Kerl JM, Ravenel JG, Nguyen SA et al. Right heart: split-bolus injection of diluted contrast medium for visualization at coronary CT angiography. Radiology 2008; 247: 356 - 364 
27 Delewi R, Zijlstra F, Piek JJ. Left ventricular thrombus formation after acute myocardial infarction. Heart 2012; 98: 1743-1749

28 Srichai MB, Junor C, Rodriguez LL et al. Clinical, imaging, and pathological characteristics of left ventricular thrombus: a comparison of contrast-enhanced magnetic resonance imaging, transthoracic echocardiography, and transesophageal echocardiography with surgical or pathological validation. American heart journal 2006; 152: 75-84
29 Mohrs OK, Nowak B, Petersen SE et al. Thrombus detection in the left atrial appendage using contrast-enhanced MRI: a pilot study. Am J Roentgenol 2006; 186: 198 - 205

30 Romero J, Cao JJ, Garcia MJ et al. Cardiac imaging for assessment of left atrial appendage stasis and thrombosis. Nat Rev Cardiol 2014; 11: $470-480$ 